\title{
EVALUACIÓN Y REHABILITACIÓN COGNOSCITIVA EN CÁNCER PEDIÁTRICO: UN ESTUDIO DE CASO
}

\section{EVALUATION AND COGNITIVE REHABILITATION IN PEDIATRIC CANCER: A CASE REPORT}

\author{
Yvonne Martínez 1,2, Erzsébet Marosi', Jaime Jorge Amador Zarco², Uriel León Jacinto ${ }^{3}$ y \\ Roberto Emmanuel Mercadillo ${ }^{4}$

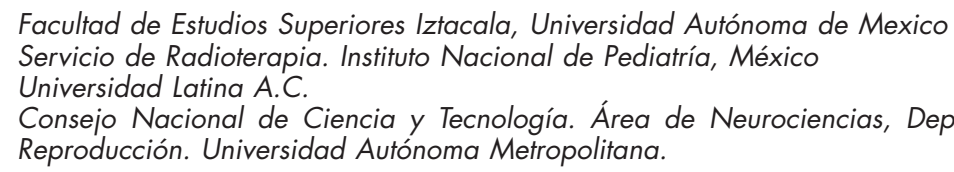

\section{Resumen}

Los niños sobrevivientes a cáncer cuya enfermedad y tratamiento involucra al Sistema Nervioso Central, presentan un patrón consistente de deficiencias neurocognoscitivas. En este artículo se describe el estudio de caso de un niño de 9 años diagnosticado con tumor de la fosa posterior sometido a cirugía, quimioterapia y radioterapia. Se aplicó la Batería Neuropsicológica Infantil en 3 momentos: posterior a la cirugía; finalizada la quimioterapia y la radioterapia y después del programa de rehabilitación cognoscitiva. Las evaluaciones neuropsicológicas después de la cirugía, quimio y radioterapia muestran deficiencias en las tareas de Habilidades Gráficas, Atención Visual y Auditiva, Porcentaje de Respuestas Correctas, Diseños Correctos con el Mínimo de Movimientos, Velocidad en la Lectura y la Escritura, Habilidades Gráficas, Codificación Visual, Memoria Verbal y Visual, Percepción Táctil, Repetición y Comprensión del Lenguaje, Habilidades Conceptuales, Fluidez Verbal y Gráfica, Precisión de la Lectura, Composición Narrativa, Conteo, Manejo Numérico y Cálculo. Después de la rehabilitación cognoscitiva observamos un incremento en el puntaje en las tareas de: Habilidades Gráficas, Memoria Verbal, Expresión y Comprensión del lenguaje, Atención Visual y Auditiva, Habilidades Conceptuales, Fluidez Verbal y Gráfica, Conteo, Manejo Numérico, Cálculo y Razonamiento Lógico Ma-
Abstract

Cancer surviving children, whose disease and treatment involves the central nervous system, show a consistent pattern of neurocognitive deficits. We report a case diagnose of a 9 years boy with posterior fossa tumor treated with surgery, chemotherapy and radiotherapy. Neuropsychological evaluation post oncological therapies and post Cognitive Remediation Program (CRP) were made to document the effect of neuropsychological rehabilitation. The results of the post oncological therapies evaluation shows deficits in Constructional Skills, Visual and Auditory Attention, Percentage of Correct Answers and Correct Designs with Minimal Movements, Speed in Reading and Writing, Graphic Skills, Visual Coding, Verbal and Visual Memory, Perception Touch, Repetition and Comprehension of Language, Conceptual Skills, Verbal and Graphics Fluency, Reading Accuracy, Narrative Composition, Count, Numerical and Calculation Management. After CRP our results illustrate an increase in the score of: Graphic Skills, Verbal Memory, Speech and Language Understanding, Conceptual Skills, Verbal and Graphics Fluency, Count, Numerical Management, Calculation and Logical Mathematical Reasoning after CRP. With the implementation of standardized measures of Evaluación Neuropsicológica

Correspondencia:

Mtra. Yvonne Martínez Chávez

Instituto Nacional de Pediatría; Servicio de Radioterapia Insurgentes Sur 3700,

Letra C, Col. Insurgentes Cuicuilco, Delegación Coyoacán, C.P 04530, México D.F.

Email: yvonnemartine19@hotmail.com 
temático. Con la implementación de las medidas normativas de la Evaluación Neuropsicológica Infantil pudimos contrastar el desempeño de nuestro paciente; el cual refleja que el programa de rehabilitación cognoscitiva potencialmente posee la eficacia suficiente para contrarrestar algunas de las secuelas neurocognoscitivas observadas después de la resección del tumor y de la radioterapia y la quimioterapia.

Palabras clave: Evaluación neuropsicológica, cáncer pediátrico, deficiencias cognoscitivas, Programa de Rehabilitación Cognoscitiva.
Infantil we could compare the performance of our patient; which suggests that the CRP has enough potential to counteract some of the neurocognitive sequelae observed after tumor resection and radio and chemotherapy.

Keywords: Neuropsychology assessment, pediatric cancer, cognitive deficits, Cognitive Remediation Program.

\section{INTRODUCCIÓN}

En la actualidad las importantes mejoras realizadas a los tratamientos utilizados en pacientes oncológicos han permitido un aumento significativo en la sobrevida de los mismos. Los niños sobrevivientes a cáncer pediátrico tienen una importante predisposición a presentar deficiencias neuropsicológicas (deficiencias en la atención, funciones ejecutivas, memoria, aprendizaje, coeficiente intelectual y rendimiento escolar), psicosociales (adaptación de la familia y el entorno al diagnóstico, pérdida de la vida normal de la familia, adaptación a los tratamientos y procedimientos médicos, abandono del trabajo por ambos o alguno de los padres, abandono de la escuela) y emocionales (miedo, inseguridad, disminución de autoestima, ansiedad, depresión, enojo, manejo del dolor) ${ }^{(1-3)}$. En lo referente a las deficiencias neuropsicológicas se ha llegado al consenso de que hay un patrón consistente de deterioro el cual incluye deficiencias en la atención sostenida, en la memoria de trabajo, velocidad del procesamiento espacial y matemático; en la auto regulación de la conducta y de las emociones ${ }^{(4-7)}$. Como consecuencia de lo anterior el rendimiento escolar de estos niños se ve significativamente afectado. En la actualidad aún existe controversia acerca del efecto de la localización del tumor y las deficiencias observadas, sin embargo dado que la mayoría de los tumores cerebrales que se presentan en edad pediátrica se ubican en la fosa posterior ha incrementado el interés por estudiar las deficiencias neuropsicológicas que se observan en pacientes con este diagnóstico. El Síndrome cerebeloso cognoscitivo-afectivo (Primero descrito en adultos y después en niños), consiste en la presencia de cambios bruscos de humor, pasividad y afecto plano con ocasional conducta desinhibida e inapropiada (impulsividad, agresividad), anormalidades en las funciones ejecutivas (planeación, flexibilidad, disminución del razonamiento abstracto, memoria de trabajo y viso-espacial). Este síndrome se asocia con anomia leve y agramatismo dando como resultado un descenso general de la función intelectual ${ }^{(8)}$.

Dado el incremento en la tasa de supervivencia de estos pacientes y el conocimiento de las secuelas neuropsicológicas que presentan (producto de los tratamientos utilizados contra el cáncer), ha surgido el interés por diseñar programas de rehabilitación cognoscitiva encaminados a disminuir las secuelas observadas. Este término se refiere a los intentos sistemáticos de mejorar el funcionamiento cognoscitivo después de presentar daño cerebral. Estos métodos involucran práctica masiva y entrenamiento por aproximación, así como otras técnicas basadas en intervenciones 
psicológicas. En los niños, el desarrollo del sistema nervioso central no se ha completado y por lo tanto no han emergido todas las funciones cognoscitivas. Las alteraciones que se detectan no van a suponer una pérdida de la función, sino una disminución en las capacidades que debe desarrollar en comparación con su grupo de iguales ${ }^{(9)}$. El cerebro del niño tiene la capacidad de recuperarse mejor de los daños focales, pero en daños generalizados el pronóstico es peor que en los adultos puesto que los niños preservan mejor las funciones básicas pero la recuperación cognoscitiva no suele ser completa ${ }^{(10)}$.

En 1998 Butler et al., re-direccionaron las técnicas de rehabilitación tradicionales utilizadas en personas con daño cerebral adquirido hacia niños que habían sido tratados por leucemia o por tumores cerebrales. Estos autores, desarrollaron un modelo tripartito que incluye técnicas y métodos de tres disciplinas: rehabilitación tradicional de daño cerebral, psicología educativa y psicología clínica. Estas terapias fueron integradas en un tratamiento llamado Programa de Rehabilitación Cognoscitiva (CRP por sus siglas en inglés). El primer caso en el que se reportaron los efectos del CRP fue de un niño que había recibido radioterapia (RT), el cual presentó mejoras en una prueba de atención sostenida después de la intervención ${ }^{(11)}$. En el 2002 Butler y Copeland realizaron un estudio piloto para probar la eficacia del CRP. Después de la rehabilitación observaron mejoras significativas en todas las medidas de atención ${ }^{(12)}$. En el 2008 Butler et al. confirmaron estos hallazgos en una población mayor, la cual después de haber recibido la rehabilitación mostró un incremento significativo en el rendimiento escolar. Con base en los resultados obtenidos, los autores proponen al CRP como un instrumento potencialmente beneficioso para contrarrestar las deficiencias cognoscitivas observadas en pacientes sobrevivientes al cáncer ${ }^{(13)}$.
El objetivo de esta investigación fue mostrar la efectividad de la aplicación del CRP, en un estudio de caso de un niño de 9 años diagnosticado con tumor en la fosa posterior, al que se le realizaron tres evaluaciones neuropsicológicas: la primera después de la resección del tumor y antes de recibir quimioterapia (QT) y radioterapia (RT) (Fase 1); la segunda al finalizar la RT y la QT (Fase 2) y la tercera concluido el CRP (Fase 3); con la finalidad de analizar los cambios en los perfiles cognoscitivos para conocer el efecto de la resección del tumor, el efecto de la RT y la QT y valorar la eficacia del CRP, así como hacer un seguimiento de los indicadores emocionales en las diferentes etapas del estudio. La hipótesis central de esta investigación fue que después de trabajar con el CRP se observarían menos secuelas neuropsicológicas asociadas a la resección del tumor y al tratamiento de RT y QT.

\section{CASO CLÍNICO}

Niño de 9 años de edad con diagnóstico de astrocitoma pilocítico en la fosa posterior, con daño en el cerebelo en la zona del vermix y a la altura del IV ventrículo. Su familia está conformada por padre, madre y hermano menor. Los padres acudieron al servicio hospitalario debido a que el paciente presentaba vómitos en propulsión matutinos, alteraciones en la marcha y aumento de sueño considerable. Después de la exploración física y estudios de gabinete y de imagen se emitió el diagnóstico. El paciente fue remitido al servicio de neurocirugía para la resección del tumor. Con el procedimiento quirúrgico se logró resecar el $80 \%$ de la totalidad del tumor. El paciente estuvo en el servicio de terapia intensiva bajo sedación durante 2 días. Al despertar mostró parálisis facial del lado derecho, dificultad para coordinar movimientos, habla lenta y de bajo volumen (acudió a rehabilitación física durante 
4 meses para mejorar la parálisis facial, la coordinación de movimientos y la marcha); 20 días después de la operación se inició el tratamiento de RT, se administró una dosis de 50.4 Gy en 28 sesiones diarias de 1.8 Gy. Al término de la RT se observaron grandes mejorías en la parálisis facial, en los problemas del lenguaje y en la marcha. Tras la RT se consolida el tratamiento con QT ambulatoria, la cual fue dividida en 6 bloques, los fármacos administrados fueron carboplatino y vincristina. Al final del mismo se observó una pérdida de la audición con mayor afección en el oído derecho, así como una neuropatía periférica tratada con GABApentina de 300mg. Durante la etapa de la QT el paciente presentó cambios en el estado de ánimo, ansiedad, depresión y problemas para dormir, para contrarrestar estos síntomas fue tratado con sertralina de $25 \mathrm{mg}$.

En cuanto a la personalidad y el estado emocional, antes de la operación el paciente mostraba necesidad de mantener orden exacerbado en su cuarto, cajones y cosas de la escuela. No le gustaba compartir sus pertenencias ni que se las movieran de lugar. En la escuela participaba constantemente, era muy estudioso, obediente y mantenía buenas relaciones, cuando se daba cuenta de que algún compañero cometía alguna falta lo externaba a la maestra. Le gustaba ganar en todas las ocasiones y se esforzaba por ser el mejor de su clase. Tenía un promedio de 9.7/10 en sus calificaciones escolares. Después de la operación mostró un comportamiento más maduro y relajado, disminuyó su interés por ser el mejor de su clase. Durante el tratamiento de RT no mostró ningún cambio, a diferencia de cuando recibió el tratamiento de QT ya que durante este le preocupaba demasiado no perder el año escolar, se mostraba muy ansioso, irritable, triste y con alteraciones del sueño. Después del tratamiento desaparecieron estos síntomas. En el transcurso de las sesiones de evaluación como en las del entrenamiento tanto el paciente como su familia mostraron una actitud muy colaborativa. El paciente siguió todas las instrucciones teniendo un trato amable, cariñoso y respetuoso. En las sesiones del entrenamiento se mostró muy motivado para realizar las actividades y para hacerlo cada vez mejor. En cuanto a su estado de ánimo se mantenía estable, contento, hacia bromas y manifestaba que le gustaban mucho las actividades.

\section{Evaluación neuropsicológica}

La Evaluación Neuropsicológica Infantil (ENI) es la primera batería neuropsicológica orientada hacia la evaluación de niños de edad escolar desarrollada y estandarizada en la población latinoamericana, en particular de México (en Guadalajara y Tijuana) y Colombia (en Manizales). Varias de las tareas que se incluyen en esta batería nacieron de planteamientos teóricos específicos y puntuales; por ejemplo, el dominio de Habilidades Espaciales, el cual está dividido en Habilidades Espaciales con contenido verbal y sin este tipo de contenido. Su estandarización recibió el rigor metodológico que requiere toda estandarización de un instrumento de evaluación. Las normas de la ENI se obtuvieron en una muestra de 788 niños de 5 a 16 años provenientes de México y Colombia, casi la mitad fueron varones, de escuelas públicas y privadas, sin antecedentes de problemas de desarrollo y/o enfermedades graves. Para poder ofrecer los datos normativos de este instrumento se llevó a cabo una serie de procedimientos de confiabilidad y validez, tales como confiabilidad de test-retest, confiabilidad entre calificadores, correlaciones con el WISC y validez interna de sus tareas. La ENI permite evaluar 9 dominios neuropsicológicos: Habilidades Construccionales, Habilidades Gráficas, Memoria a corto plazo y diferida en su modalidad Verbal y no Verbal, Habilidades Perceptuales (Visuales, 
Auditivas y Táctiles), Lenguaje que incluye la Expresión, Comprensión y Repetición, Habilidades Metalingüísticas, Habilidades Espaciales, Atención (Visual y Auditiva), Habilidades Conceptuales, Funciones Ejecutivas que son los propios de una evaluación neuropsicológica estándar. Se incluyen además, 3 áreas académicas: Lectura, Escritura y Aritmética. Los dominios están divididos en subdominios y tareas cuyo número varía entre ellos; por ejemplo, el dominio de Lenguaje está dividido en tres subdominios: Repetición, Expresión y Comprensión. A su vez, la tarea de repetición consta de 4 tareas; Repetición de sílabas, de palabras, no palabras y oraciones. En total son 13 dominios, 36 subdominios y 79 tareas. En la tabla 1 se muestra el esquema de organización de la $\mathrm{ENI}^{(14-17)}$.

Las puntuaciones finales de la ENI se obtienen en percentiles, cuyos valores se ajustan a los siguientes rangos cuantitativos: $\leq 2=$ extremadamente bajo; $3-10=$ bajo; $11-25=$ promedio bajo; $26-75=$ promedio. Los valores por arriba del percentil 75 se consideran superiores.

La evaluación de los indicadores emocionales se realizó a través del dibujo de la figura humana (DFH) utilizando el método de Koppitz (1976), el cual analiza los DFH en función de dos tipos de signos objetivos abarcando un rango de edad de los 5 a los 12 años de edad ${ }^{(18-19)}$. Un primer conjunto de signos se considera que está primariamente relacionado con la edad y el nivel de maduración; a estos signos los denominamos Indicadores Evolutivos. Un segundo conjunto de signos está primariamente relacionado con las actitudes y preocupaciones del niño; a estos signos los denominamos Indicadores Emocionales. Los Indicadores Emocionales (IE) son signos clínicos que reflejan actitudes y características subyacentes de los niños en el momento de realizar sus DFH (ver la tabla 2). Los IE no son mutuamente excluyentes (ver la tabla 3); varios IE revelan sentimien- tos y preocupaciones iguales o similares, y una misma actitud puede ser expresada por diversos IE agrupados en categorías ${ }^{(20)}$.

\section{Programa de Rehabilitación Cognoscitiva}

La primera parte corresponde a la rehabilitación de la atención para lo cual se utiliza el Attention Process Training (APT) material desarrollado por Sohlberg y Mateer (2001). Este programa utiliza la regla $50 \%-80 \%$ con la cual el niño debe obtener un mínimo de $50 \%$ de aciertos de la tarea para poder continuar, en caso de que el niño no cumpla esta regla se utiliza una tarea menos compleja. Una vez que el niño alcanza el $80 \%$ de aciertos se le administra una tarea de mayor dificultad ${ }^{(21)}$. Por ejemplo una tarea consiste en decirle al niño una letra del abecedario y el debe decir la que va antes y la que va después, se cuantifica el tiempo en el que realiza la tarea así como los aciertos y los errores para dar retroalimentación al final de la sesión. La parte de psicología educativa corresponde a estrategias metacognoscitivas que se le enseñan al niño para que pueda realizar con mejores resultados las tareas. Se le enseñan estrategias que lo preparan para realizar la tarea, estrategias que debe emplear durante y después de la realización de la tarea. Si durante alguna sesión el niño utiliza una estrategia en particular y esta le ayuda a mejorar su rendimiento en la tarea, esa estrategia formará parte del repertorio de estrategias exitosas de aprendizaje. La tercera parte del programa corresponde al aspecto clínico. Aquí se incorporan varios elementos propuestos por Meichenbaum (1993), enfocados a lograr que el niño convierta sus luchas internas en aspectos positivos $^{(22)}$. Se le enseña a hablar acerca de las situaciones que le generan estrés, angustia o que le son sumamente complicadas. Se refuerzan los puntos clave del aprendizaje y se trabaja para lograr que el niño tenga control interno sobre sí mismo. Se dirige al niño para que se convierta en su "mejor amigo" 


\section{Tabla 1. Tareas de la Evaluación Neuropsicológica Infantil(17)}

\begin{tabular}{|c|c|}
\hline TAREAS & TAREAS \\
\hline 1. Habilidades construccionales & 8. Escritura \\
\hline 1.1. Construcción con palillos (8) & 8.1. Precisión \\
\hline 1.2. Habilidades gráficas & 8.1.1. Escritura del nombre (2) \\
\hline 1.2.1. Copia de figuras (12) & 8.1.2. Dictado de sílabas (8) \\
\hline 1.2.2. Dibujo de la figura humana (20) & 8.1.3. Dictado de palabras (8) \\
\hline 1.2.3. Copia de la figura compleja (12-15) & 8.1.4. Dictado de no palabras (8) \\
\hline 2. Memoria (codificación) & 8.1.5. Dictado de oraciones (20) \\
\hline 2.1. Memoria verbal-auditiva & 8.1.6. Copia de un texto \\
\hline 2.1.1. Lista de palabras $(36-48)$ & 8.1.7. Recuperación escrita \\
\hline 2.1.2.Recuerdo de una historia (15) & 8.2. Composición narrativa \\
\hline 2.2. Memoria visual-Lista de figuras $(36-48)$ & 8.2.1. Coherencia narrativa $(7)$ \\
\hline 3. Habilidades Perceptuales & 8.2.2. Longitud de la producción narrativa \\
\hline 3.1. Percepción táctil & 8.3. Velocidad \\
\hline 3.1.1. Mano derecha (8) & 8.3.1. En la copia de un texto \\
\hline 3.1.2. Mano izquierda (8) & 8.3.2. En la recuperación escrita \\
\hline 3.2. Percepción visual & 9. Aritmética \\
\hline 3.2.1. Imágenes sobrepuestas (16) & 9.1. Conteo (8) \\
\hline 3.2.2. Imágenes borrosas (10) & 9.2. Manejo numérico \\
\hline 3.2.3. Cierre visual (8) & 9.2.1. Lectura de números (8) \\
\hline 3.2.4. Reconocimiento de expresiones (8) & 9.2.2. Dictado de números (8) \\
\hline 3.2.5. Integración de objetos (8) & 9.2.3. Comparación de números (8) \\
\hline 4. Memoria (evocación diferida) & 9.2.4. Ordenamiento de cantidades (8) \\
\hline 4.1. Evocación de estímulos auditivos. & 9.3. Cálculo \\
\hline 4.1.1. Espontánea de la lista de palabras (9-12) & 9.3.1. Serie directa (8) \\
\hline 4.1.2. Por claves $(9-12)$ & 9.3.2. Serie inversa ( 8 ) \\
\hline 4.1.3. Reconocimiento (18-24) & 9.3.3. Cálculo mental (12) \\
\hline 4.1.4. De la historia (15) & 9.3.4. Cálculo escrito (14) \\
\hline 4.2. Evocación de estímulos visuales. & 9.4. Razonamiento lógico-matemático \\
\hline 4.2.1. Figura compleja $(12-15)$ & 9.4.1. Problemas numéricos (8) \\
\hline 4.2.2. Espontánea de la lista de figuras (9-12) & 10. Habilidades espaciales \\
\hline 4.2.3. Por claves $(9-12)$ & 10.1. Comprensión derecha - izquierda (8) \\
\hline 4.2.4. Reconocimiento (18-24) & 10.2. Expresión derecha - izquierda (8) \\
\hline 5. Lenguaje & 10.3. Dibujos desde ángulos diferentes (8) \\
\hline 5.1. Repetición & 10.4. Orientación de líneas (8) \\
\hline 5.1.1. Sílabas (8) & 10.5. Ubicación de coordenadas (8) \\
\hline 5.1.2. Palabras (8) & 11. Atención \\
\hline 5.1.3. Repetición de no palabras (8) & 11.1. Atención visual \\
\hline 5.1.4. Repetición de oraciones (8) & 11.1.1. Cancelación de dibujos (44) \\
\hline 5.2. Expresión & 11.1.2. Cancelación de letras (82) \\
\hline 5.2.1. Denominación de imágenes (15) & 11.2. Atención auditiva \\
\hline 5.2.2. Coherencia narrativa (6) & 11.2.1. Dígitos en progresión (8) \\
\hline 5.2.3. Longitud de la expresión (307) & 11.2.2. Dígitos en regresión (7) \\
\hline 5.3. Comprensión & 12. Habilidades conceptuales \\
\hline 5.3.1. Designación de imágenes (15) & 12.1. Similitudes (16) \\
\hline 5.3.2. Seguimiento de instrucciones (10) & 12.2. Matrices (8) \\
\hline 5.3.3. Comprensión del discurso (10) & 12.3. Problemas aritméticos (8) \\
\hline 6. Habilidades metalingüísticas & 13. Funciones ejecutivas \\
\hline 6.1. Síntesis fonémica (8) & 13.1. Fluidez verbal \\
\hline 6.2. Conteo de sonidos (8) & 13.1.1. Fluidez semántica (28) \\
\hline 6.3. Deletreo (8) & 13.1.2. Fluidez fonológica (28) \\
\hline 6.4. Conteo de palabras (8) & 13.2. Fluidez gráfica \\
\hline 7. Lectura & 13.2.1. Fluidez semántica (35) \\
\hline 7.1. Precisión & 13.2.2. Fluidez no-verbal (35) \\
\hline 7.1.1. Sílabas (8) & 13.3. Flexibilidad cognitiva \\
\hline 7.1.2. Palabras (8-11) & 13.3.1. Número de ensayos administrados \\
\hline 7.1.3. No palabras (8) & 13.3.2. Total de errores \\
\hline 7.1.4. Oraciones (10) & 13.3.3. Porcentaje de errores \\
\hline 7.1.5. Lectura en voz alta de un texto & 13.3.4. Número de categorías \\
\hline 7.2. Comprensión & 13.3.5. Incapacidad para mantener la organización \\
\hline 7.2.1. Oraciones (10) & 13.3.6. Número de respuestas perseverativas \\
\hline 7.2.2. Lectura en voz alta de un texto & 13.3.7. Porcentaje de respuestas perseverativas \\
\hline 7.2.3. Lectura silenciosa de un texto & 13.4. Planeación y Organización \\
\hline 7.3. Velocidad & 13.4.1. Diseños correctos (11) \\
\hline 7.3.1. Lectura en voz alta & 13.4.2. Movimientos Realizados (11) \\
\hline 7.3.2. Lectura silenciosa de un texto de un texto & 13.4.3. Diseños correctos con el mínimo de movimientos (11) \\
\hline
\end{tabular}

en lugar de ser su "peor enemigo". Todo dentro del marco cognitivo conductual[ ${ }^{(22)}$. Se trabajó un total de 13 sesiones. El programa está dirigido a desarrollar métodos cognoscitivos conductuales que eviten que el niño pierda la concentración incluso si está en un ambiente con varios distractores. La decisión de implementar este programa 


\section{Tabla 2. Listado de los Indicadores emocionales válidos y no válidos (no se consideran en el análisis) del dibujo de la figura humana ${ }^{(20)}$}

\begin{tabular}{ll}
\hline \multicolumn{1}{c}{ Listado de Indicadores Emocionales válidos y no válidos } \\
\hline \multicolumn{1}{c}{ Indicadores Emocionales Válidos } & \multicolumn{1}{c}{ Indicadores Emocionales no Válidos } \\
\hline Integración pobre de las partes de la figura & Cabeza grande \\
Sombreado de la cara & Ojos vacíos u ojos que no ven \\
Sombreado del cuerpo y/o extremidades & Mirada de reojo \\
Sombreado de las manos y/o cuello & Manos Ocultas \\
Asimetría grosera de las extremidades & Figura interrumpida por el borde de la hoja \\
Figura inclinada & Línea de base o pasto \\
Figura pequeña & Sol o luna \\
Figura grande & Líneas fragmentadas o esbozadas \\
Transparencia & \\
Cabeza pequeña & \\
Ojos bizcos o desviados & \\
Dientes & \\
Brazos cortos & \\
Brazos largos & \\
Brazos pegados al cuerpo & \\
Manos grandes & \\
Manos seccionadas u omitidas & \\
Piernas juntas & \\
Figura desnuda, genitales & \\
Figura monstruosa o grotesca & \\
Dibujo espontáneo de tres o más figuras & \\
Nubes, lluvia, nieve, pájaros volando & \\
Omisión de los ojos & \\
Omisión de la nariz & \\
Omisión de la boca & \\
Omisión del cuerpo & \\
Omisión de los brazos & \\
Omisión de las piernas & \\
Omisión de los pies & \\
Omisión del cuello & \\
\hline
\end{tabular}

en nuestro estudio se tomó con base en que el CRP es el método mejor descrito en la literatura y el que ha arrojado resultados favorables sobre los pacientes; además de ser un sistema que no se enfoca a una sola área de acción, sino que, tiene una visión integradora que permite utilizar varios elementos para promover la rehabilitación de los niños.

\section{RESULTADOS}

\section{Funciones cognoscitivas}

Para una mejor visualización de los resultados de la ENI, se presentan los valores obtenidos en las tres evaluaciones agrupados en dominios.
FASE 1

Los resultados obtenidos durante la primera fase muestran que en el dominio de Funciones Cognoscitivas la presencia del tumor solamente afecto las tareas de Habilidades Gráficas y Atención Visual ya que éstas se ubicaron por debajo de lo normal, para el resto de las tareas los puntajes obtenidos por el niño se ubican dentro del rango de normalidad o por arriba de éste (Figura 1).

\section{FASE 2}

En la fase 2 después de la RT y QT se observó un incremento en las puntuaciones logradas en Construcción con Palillos, Codifi- 
Tabla 3: Categorías de los Indicadores emocionales del dibujo de la figura humana(20)

\begin{tabular}{|c|c|c|}
\hline \multicolumn{3}{|c|}{ Categorías de los Indicadores Emocionales } \\
\hline Categoría & Definición & Indicadores Emocionales \\
\hline Impulsividad & $\begin{array}{l}\text { Tendencia a actuar espontáneamente, casi sin premeditación o pla- } \\
\text { neación; a mostrar baja tolerancia a la frustración, control interno débil, } \\
\text { inconsistencia; a ser expansivo y a buscar gratificación inmediata. La } \\
\text { impulsividad se relaciona por lo común, con el temperamento de los } \\
\text { jóvenes, con inmadurez (o ambos) o con afección neurológica. }\end{array}$ & $\begin{array}{l}\text { Integración pobre de las partes de la figura } \\
\text { Asimetría grosera de las extremidades } \\
\text { Figura grande } \\
\text { Transparencia } \\
\text { Omisión del cuello }\end{array}$ \\
\hline $\begin{array}{l}\text { Inseguridad } \\
\text { Sentimientos de } \\
\text { inadecuación }\end{array}$ & $\begin{array}{l}\text { Implica un auto concepto bajo, falta de seguridad en sí mismo, } \\
\text { preocupación acerca de la adecuación mental, sentimientos de im- } \\
\text { potencia y una posición insegura. El niño se considera como un } \\
\text { extraño, no lo suficientemente humano, o como una persona ridí- } \\
\text { cula que tiene dificultades para establecer contacto con los demás. }\end{array}$ & $\begin{array}{l}\text { Figura inclinada } \\
\text { Cabeza pequeña } \\
\text { Manos seccionadas u omitidas } \\
\text { Figura monstruosa o grotesca } \\
\text { Omisión de los brazos } \\
\text { Omisión de las piernas } \\
\text { Omisión de los pies } \\
\end{array}$ \\
\hline Ansiedad & $\begin{array}{l}\text { Tensión o inquietud de la mente con respecto al cuerpo (ansiedad cor- } \\
\text { poral), a las acciones, al futuro; preocupación, inestabilidad, aflicción; } \\
\text { estado prolongado de aprensión. }\end{array}$ & $\begin{array}{l}\text { Sombreado de la cara } \\
\text { Sombreado del cuerpo y / o extremidades } \\
\text { Sombreado de las manos y / o cuello } \\
\text { Piernas juntas } \\
\text { Nubes, lluvia, nieve, pájaros volando } \\
\text { Omisión de los ojos } \\
\end{array}$ \\
\hline $\begin{array}{l}\text { Apocamiento, } \\
\text { Timidez: }\end{array}$ & $\begin{array}{l}\text { Conducta retraída, cautelosa y reservada; falta de seguridad en sí mis- } \\
\text { mo; tendencias a avergonzarse, tendencia a atemorizarse fácilmente, a } \\
\text { apartarse de las circunstancias difíciles o peligrosas. }\end{array}$ & $\begin{array}{l}\text { Figura pequeña } \\
\text { Brazos cortos } \\
\text { Brazos pegados al cuerpo } \\
\text { Omisión de la nariz Omisión de la boca } \\
\text { Omisión de los pies } \\
\end{array}$ \\
\hline $\begin{array}{l}\text { Ira } \\
\text { Agresividad }\end{array}$ & $\begin{array}{l}\text { Disgusto, resentimiento, exasperación o indignación; actitud ofensiva } \\
\text { en general; sentimientos de venganza hacia quienes son percibidos } \\
\text { como causantes de agravio; ataques verbales o físicos; furia resultante } \\
\text { de la frustración. }\end{array}$ & $\begin{array}{l}\text { Ojos bizcos o desviados } \\
\text { Dientes } \\
\text { Brazos largos } \\
\text { Manos grandes } \\
\text { Figura desnuda, genitales }\end{array}$ \\
\hline Robo & $\begin{array}{l}\text { Tomar cosas o tener en posesión cosas que pertenecen a otros sin su } \\
\text { permiso. }\end{array}$ & $\begin{array}{l}\text { Sombreado del cuerpo y / o extremidades } \\
\text { Sombreado de las manos y / o cuello } \\
\text { Figura grande } \\
\text { Transparencia } \\
\text { Cabeza pequeña } \\
\text { Manos grandes } \\
\text { Piernas juntas } \\
\text { Omisión del cuerpo } \\
\text { Omisión de los brazos } \\
\text { Omisión del cuello } \\
\end{array}$ \\
\hline $\begin{array}{l}\text { Mal Desempeño } \\
\text { escolar }\end{array}$ & $\begin{array}{l}\text { Bajo rendimiento académico, bajas calificaciones, dificultades en la } \\
\text { lectura, escritura y matemáticas. }\end{array}$ & $\begin{array}{l}\text { Integración pobre de las partes de la figura } \\
\text { Figura pequeña } \\
\text { Figura monstruosa o grotesca } \\
\text { Dibujo espontáneo de tres o más figuras } \\
\text { Omisión de la boca } \\
\text { Omisión del cuerpo } \\
\text { Omisión de los brazos }\end{array}$ \\
\hline
\end{tabular}

cación Verbal, Expresión del Lenguaje, Habilidades Metalingüísticas y en Atención Visual (a pesar de la mejoría los puntajes de esta tarea no alcanzaron el rango de normalidad). No obstante, se observaron puntajes más bajos en las tareas de Habilidades Gráficas, Codificación Visual, Evocación Verbal, Percepción Táctil, Repetición del Lenguaje, Comprensión del Lenguaje, Atención Auditiva, Habilidades Conceptuales, cabe mencionar que a pesar de la disminución de los puntajes todas las tareas se ubican dentro del rango de normalidad. La única tarea que se ubica debajo del rango de normalidad después de la RT y la QT es la de Evocación visual (Figura 1).

\section{FASE 3}

Después del CRP se observó un incremento en los puntajes de Habilidades 


\section{Figura 1. Dominio de Funciones Cognoscitivas. Promedio de las puntuaciones obtenidas en las tres evaluaciones}

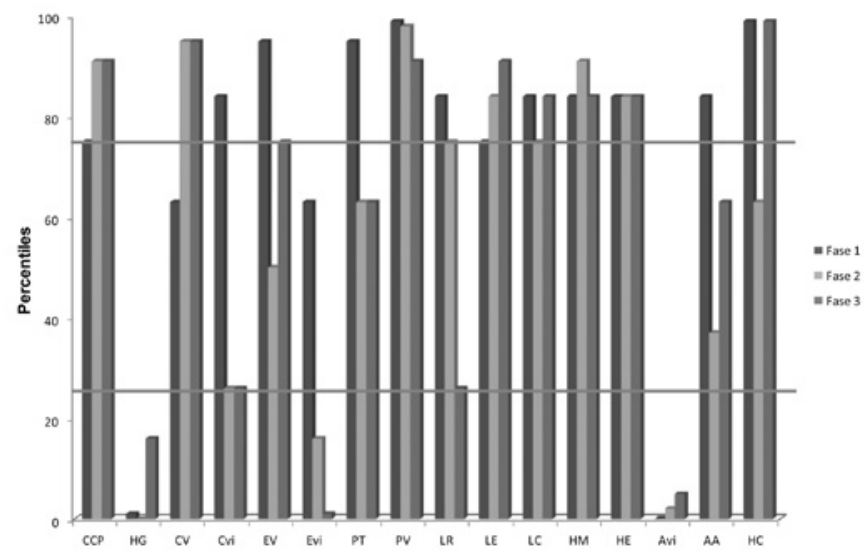

Fase 1 (Antes de la Radioterapia y Quimioterapia); Fase 2 (Después de la Radioterapia y Quimioterapia); Fase 3 (Después del Programa de Rehabilitación Cognoscitiva) CCP: Construcción con Palillos, HG: Habilidades Gráficas, CV: Codificación Verbal, Cvi: Codificación Visual, EV: Evocación Verbal, Evi: Evocación Visual, PT: Percepción Táctil, PV: Percepción Visual, LR: Lenguaje Repetición, LE: Lenguaje Expresión, LC: Lenguaje Comprensión), HM: Habilidades Metalingüísticas, HE: Habilidades Espaciales, Avi: Atención Visual, AA: Atención Auditiva, HC: Habilidades Conceptuales. Las líneas rojas indican los valores ubicados dentro del rango promedio (percentil 26-75) de acuerdo a las normas de la Evaluación Neuropsicológica Infantil.

Gráficas y Atención Visual, pero no el suficiente para alcanzar el rango de normalidad. Se observaron mayores puntajes en la fase 3 en comparación con la fase 2 en las tareas de Evocación Visual, Expresión del Lenguaje, Comprensión del Lenguaje, Atención Auditiva, y Habilidades Conceptuales. La tarea de Expresión del Lenguaje obtuvo puntajes más altos en la fase 3 en comparación con los obtenidos en la fase 1. A su vez se observaron puntajes más bajos en las tareas de Evocación Visual, Repetición del Lenguaje y Habilidades Metalingüísticas (Figura 1).

\section{Funciones ejecutivas}

\section{FASE 1}

En el dominio de las Funciones Ejecutivas, la presencia del tumor afectó las tareas de Porcentaje de Respuestas Correctas y Diseños Correctos con el Mínimo de Movimientos las cuales se ubican por debajo de lo normal. En el resto de las tareas, los puntajes se ubican dentro y por arriba de lo normal (ver la figura 2).

\section{FASE 2}

Después de la RT y QT se observó una gran mejoría en los puntajes obtenidos en la tarea de Porcentaje de Respuestas Correctas, Porcentaje de Respuestas Perseverativas y Número de Categorías; sin embargo se observaron puntajes más bajos en la tarea de Fluidez Verbal, Fluidez Gráfica y Diseños Correctos con el Mínimo de Movimientos. El resto de las tareas no mostraron cambios (ver la figura 2).

\section{FASE 3}

Al finalizar el CRP se observó mejoría en la ejecución del sujeto al compararla con la ejecución de la fase 2 en las tareas de Fluidez Verbal y en Fluidez Gráfica. En la tarea de Diseños Correctos con el Míni- 
mo de Movimientos se observaron puntajes más bajos. En el resto de las tareas se mantuvieron los puntajes de la fase 2 (Figura 2).

\section{Habilidades de rendimiento académico}

\section{FASE 1}

En el dominio de Habilidades y Rendimiento Académico, en la fase 1 el niño logró puntajes dentro de la normalidad o por arriba de ésta en la mayoría de las tareas. La tarea de Velocidad de Lectura y Escritura se ubicó muy por debajo de lo normal en las 3 fases (ver la figura 3 ).

\section{FASE 2}

Después de la RT y QT se observó una ligera mejoría en la tarea de velocidad de Lectura, no obstante en las tareas de Precisión de la Lectura, Coherencia Narrativa, Conteo, Manejo Numérico y Cálculo se observaron puntajes más bajos (ver la figura 3).

\section{FASE 3}

Se observaron mejores puntajes en la fase 3 en las tareas de Conteo, Manejo Numérico, Cálculo y Razonamiento Lógico Matemático, sin embargo los puntajes de la tarea de Coherencia Narrativa fueron menores (ver la figura 3).

\section{Indicadores emocionales}

\section{FASE 1}

En el dibujo que corresponde a la evaluación en la fase 1 se observa una figura grande, grotesca; con pobre integración de sus partes, brazos largos y pegados al cuerpo con piernas, pies y manos omitidas. Estos son indicadores de impulsividad, inseguridad y sentimientos de inadecuación, ansiedad y timidez.

\section{Figura 2. Dominio de Funciones Ejecutivas. Promedio de las puntuaciones obtenidas en las tres evaluaciones}

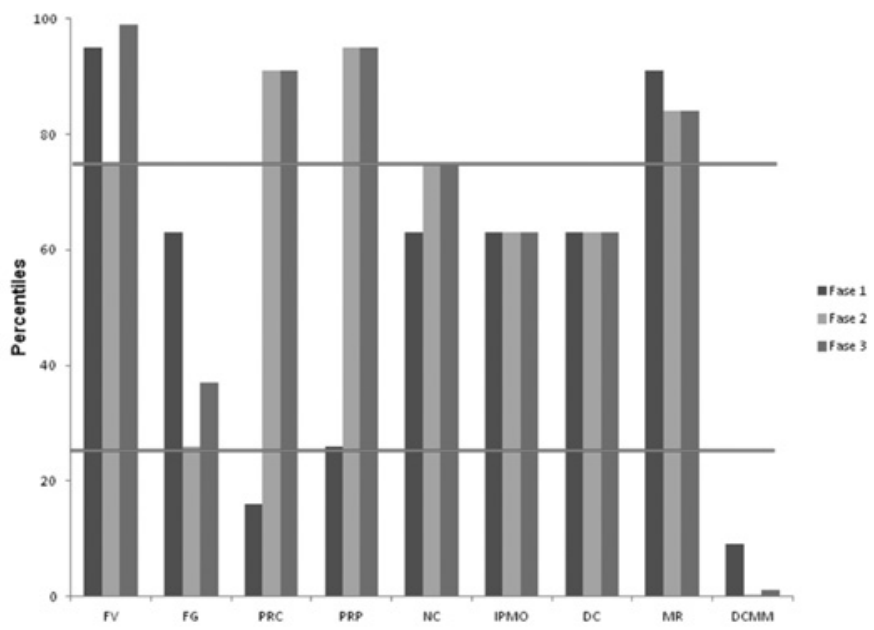

Fase 1 (Antes de la Radioterapia y Quimioterapia); Fase 2 (Después de la Radioterapia y Quimioterapia); Fase 3 (Después del Programa de Rehabilitación Cognoscitiva) CCP. FV: Fluidez Verbal, FG: Fluidez Gráfica, PRC: Porcentajes de Respuestas Correctas, PRP: Porcentaje de Respuestas Perseverativas, NC: Número de Categorías, IPMO: Incapacidad para Mantener la Organización, DC: Diseños Correctos, MR: Movimientos Realizados, DCMM: Diseños Correctos con el Mínimo de Movimientos. Las líneas rojas indican los valores ubicados dentro del rango promedio (percentil 26-75) de acuerdo a las normas de la Evaluación Neuropsicológica Infantil. 


\section{Figura 3. Dominio de Habilidades de Rendimiento Académico. Promedio de las puntuaciones obtenidas en las tres evaluaciones}

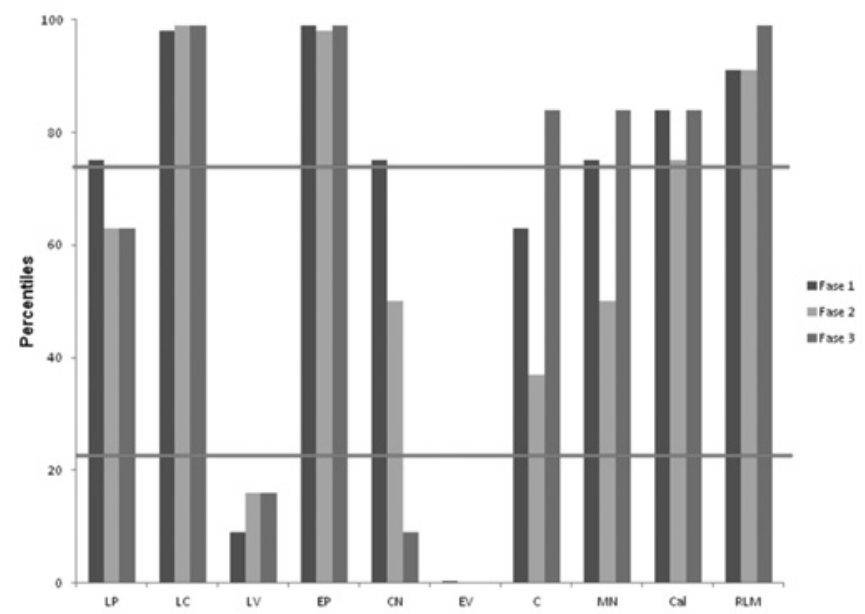

Fase 1 (Antes de la Radioterapia y Quimioterapia); Fase 2 (Después de la Radioterapia y Quimioterapia); Fase 3 (Después del Programa de Rehabilitación Cognoscitiva) CCP. LP: Lectura Precisión, LC: Lectura Comprensión, LV: Lectura Velocidad, EP: Escritura Precisión, CN: Coherencia Narrativa, EV: Escritura Velocidad, C: Conteo, MN: Manejo Numérico, Cal: Cálculo, RLM: Razonamiento Lógico-Matemático. Las líneas rojas indican los valores ubicados dentro del rango promedio (percentil 26-75) de acuerdo a las normas de la Evaluación Neuropsicológica Infantil.

\section{FASE 2}

En el dibujo que corresponde a la evaluación de la Fase 2 se observa una cabeza grande, con omisión de cuello, cuerpo, brazos, piernas, manos y pies. Estos son indicadores de inseguridad y sentimientos de inadecuación, timidez, ansiedad y mal desempeño escolar.

\section{FASE 3}

El dibujo de la figura humana correspondiente a la fase 3 muestra un tamaño normal de la figura y salvo el sombreado del cabello que denota ansiedad no se observa ningún otro indicador emocional, no se observan omisiones, mala integración o distorsión de la figura (ver la figura 4).

\section{DISCUSIÓN}

La hipótesis central de este trabajo sostiene que los puntajes obtenidos en la fase 3 mostrarían un mejor desempeño en comparación con los resultados obtenidos en la fase 1 y la fase 2. Se observaron puntajes más altos en la última evaluación en las tareas de Construcción con PaliIlos, Codificación Verbal, Expresión del Lenguaje, Fluidez Verbal, Porcentaje de Respuestas Correctas y Respuestas Perseverativas, Número de Categorías, Conteo, Manejo Numérico y Razonamiento Lógico Matemático. En cuanto a la comparación entre la fase 2 y 3 se observaron puntajes más elevados en las tareas de Habilidades Gráficas, Memoria Verbal, Expresión y Comprensión del Lenguaje, Atención Auditiva, Habilidades Conceptuales, Fluidez Verbal y Gráfica, Conteo, Manejo Numérico, Cálculo y Razonamiento Lógico Matemático, aunado a que los padres reportaron mejoría en la conducta tanto en casa como en la escuela (Mayor flexibilidad y control emocional). Los resultados obtenidos en la fase 3 en estas tareas prueban nuestra hipótesis. Con la implementación de las medidas normativas de la ENI pudimos contrastar el desempeño de nuestro 
paciente; el cual refleja que el CRP potencialmente posee la eficacia suficiente para contrarrestar algunas de las secuelas neurocognoscitivas observadas después de la resección del tumor y de la RT y la QT. Es sabido que la rehabilitación del daño cerebral es una tarea sumamente demandante y que requiere del máximo esfuerzo del paciente y del terapeuta. Este proceso es particularmente complejo en pacientes oncológicos, debido a que sus cerebros se encuentran sanando (gliosis, calsificación y apoptosis) y en desarrollo al mismo tiempo, por lo tanto es crucial la estimulación que reciban de su entorno.

Anteriormente se consideraba que la rehabilitación del daño cerebral adquirido se caracterizaba por mostrar efectos pequeños y modestas mejoras en la cognición, educación, conducta y habilidades sociales ${ }^{(23-25)}$; afortunadamente esta visión ha cambiando; recientemente los reportes en la literatura muestran que pacientes con daño cerebral adquirido que han sido sometidos a programas de rehabilitación cognoscitiva muestran mejorías después de las intervenciones ${ }^{(26,27)}$.

Este prometedor panorama también se ha extendido al área de oncología pediátrica, ya que se han reportado efectos benéficos después de la aplicación de programas de rehabilitación cognoscitiva ${ }^{(28)}$; nuestros resultados concuerdan con estos reportes ya que después de $1 / 2$ año de entrenamiento observamos mejorías en los puntajes obtenidos en la fase 3 en comparación con los obtenidos en la fase 2, especialmente en el dominio de funciones cognoscitivas y ejecutivas, además se observaron mejorías conductuales, específicamente en la irritabilidad, ansiedad y problemas de sueño. Sin embargo, en las tareas de Evocación Visual, Percepción Visual, Repetición del Lenguaje, Habilidades Metalingüísticas y Coherencia Narrativa se obtuvieron puntajes más bajos en relación a los obtenidos en la fase 2 , cabe señalar que en el entrenamiento no se incluyeron actividades encaminadas a reforzar estas tareas y solamente se ubican por debajo de lo normal las tareas de Evocación Visual y Coherencia Narrativa.

En el resto de las tareas no se observó variabilidad en los puntajes, lo cual es un resultado alentador ya que se ha reportado que 6 meses después de la resección del tumor y de la RT y QT se comienza a ver un deterioro cognosctivo generalizado en estos niños ${ }^{(29)}$. La variabilidad de los Indicadores Emocionales concuerda con la evolución neuropsicológica ya que se observaron patrones de cambio similares; en la Fase 1 y 2 los cuales mostraron un mayor número de rasgos de ansiedad, inseguridad y timidez; en comparación con los rasgos observados en la fase 3 los cuales muestran un mejor estado emocional mismo que correlaciona con mayores capacidades intelectuales. Estos resultados sugie-

\section{Figura 4. Dibujo de la figura humana fase 1 , fase 2 y fase 3}

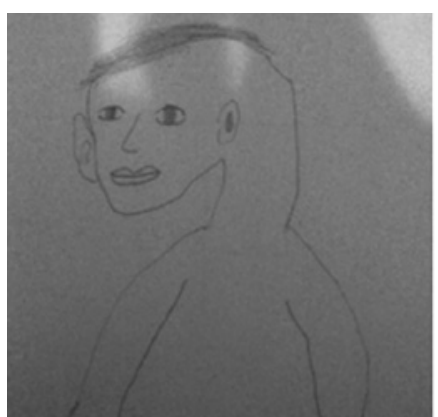

Fase 1

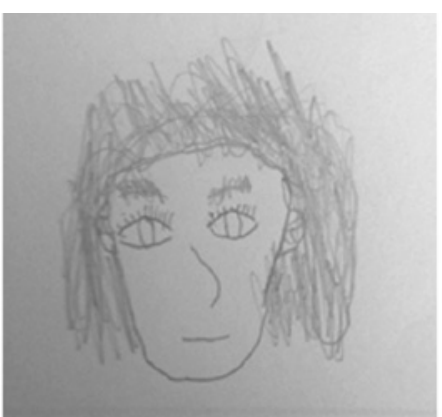

Fase 2

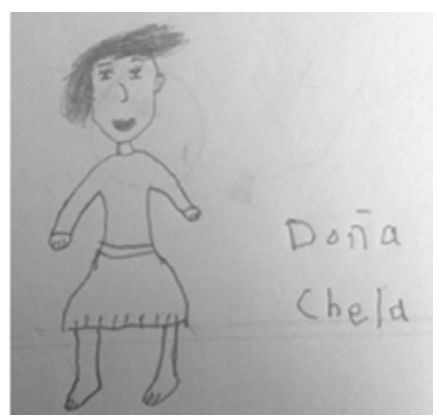

Fase 3 
ren la posibilidad de un futuro prometedor en la rehabilitación de estos pacientes.

Nos espera la tarea de sistematizar las evaluaciones neuropsicológicas realizadas a estos pacientes considerando no solamente los aspectos cognoscitivos si no también los emocionales, conductuales y de calidad de vida, así como tomar en cuenta otros factores que pueden potenciar el efecto benéfico del tratamiento, tales como la participación de los padres de familia y de los profesores de manera sistematizada e incluir en los protocolos al mayor número de participantes que sea posible; esto con la finalidad de mejorar la metodología de los tratamientos de rehabilitación ya que estos deben considerar aspectos físicos, cognoscitivos, emocionales, conductuales y sociales para que los resultados se encaminen a generar una mejor calidad de vida. Así, con la implementación de herramientas educativas, cognoscitivas y conductuales el paciente logrará respuestas más adaptativas y una mayor capacidad de autorregulación cognoscitiva y emocional ${ }^{(21)}$. Lamentablemente en nuestro país son muy pocos los servicios de salud pública que dedican recursos para generar estrategias de rehabilitación.

\section{REFERENCIAS BIBLIOGRÁFICAS}

1. Hoppe-Hirsch E, Brunet L, Laroussinie F, Cinalli G, Pierre-Kahn A, Renier D, et al. Intellectual outcome in children with malignant tumors of the posterior fossa: Influence of the field of irradiation and quality of surgery. Childs Nerv Sys 1995;11:3405. discussion 345-6.

2. Mulhern RK, Merchant TE, Gajjar A, Reddick WE, Kun LE. Late neurocognitive sequelae in survivors of brain tumours in childhood. Lancet Oncol 2004;5:399-408.

3. Ris MD, Packer R, Goldwein J, Jones-Wallace D, Boyett JM. Intellectual outcome after reduced-dose radiation therapy plus adjuvant chemotherapy for medulloblas- toma: A Children's Cancer Group study. J Clin Oncol 2001;19:3470-3476.

4. Butler RW, Hill JM, Steinherz PG, Meyers PA, Finlay JL. Neuropsychologic effects of cranial irradiation, intrathecal methotrexate, and systemic methotrexate in childhood cancer. J Clin Oncol 1994;12:2621-9.

5. Butler RW, Kerr M, Marchand A. Attention and executive functions following cranial irradiation in children. J Inter Neuropsychol Soc 1999;5:108.

6. Mulhern, R.; Butler, R. Neuropsychological late effects. In: Brown, RT., editor. Comprehensive handbook of childhood cancer and sickle cell disease: A biopsychosocial approach. New York: Oxford University Press; 2006. p. 262-78.

7. Spiegler BJ, Bouffet E, Greenberg ML, Rutka JT, Mabbott DJ. Change in neurocognitive functioning after treatment with cranial radiation in childhood. J Clin Oncol 2004;22:706-13.

8. Carrizoza JM, Cornejo OW. Cerebelo: más allá de la coordinación motora Anatomía y conexiones del cerebelo. IATREIA 2003;16:2-183-7.

9. Bernabeu Verdu J, López Luengo B, Fournier del Castillo C, Cañete Nieto A, Suárez Rodríguez J, Castel Sánchez V. Attention process training application within an intervention project on attentional processes in children with cancer Rev Neurol 2004;38:482-6.

10. Kolb B. Brain plasticity and behavior. Hillsdale, NJ: Lawrence Erlbaum Associates,Inc., 1995.

11. Butler, R. W. Attentional processes and their remediation in childhood cancer. Med Pediatr Oncol;1998;30 (Suppl. 1): 75-78.

12. Butler RW, Copeland DR. Attentional processes and their remediation in children treated for cancer: A literature review and the development of a therapeutic approach. J Inter Neuropsychol Soc 2002;8, 113-24

13. Butler RW, Copeland DR, Fairclough DL, Mulhern RK, Katz ER, Kazak AE, et al. A Multicenter, randomized clinical trial of a cognitive remediation program for child- 
hood survivors of a pediatric malignancy. J Consul Clin Psychol 2008;76:367-78. Doi:10.1037/0022-006X.76.3.367

14. Matute $\mathrm{E}$, Chamorro $\mathrm{Y}$, Inozemtseva $\mathrm{O}$, Barrios O, Rosselli M, Ardila A. Efecto de la edad en una tarea de planificación y organización ('pirámide de México') en escolares. Rev Neurol 2008;47:61-70.

15. Matute E, Rosselli M, Ardila A, Morales L. Verbal and non-verbal fluency in Spanish speaking children. Dev Neuropsychol 2004;26:647-60.

16. Matute E, Roselli M, Ardila A, OstroskySolís F. Evaluación Neurosicológica Infantil. México: Ed. Manual Moderno, 2007.

17. Matute E, Inozemtseva O, González Reyes AL, Chamorro Y. La Evaluación Neuropsicológica Infantil (ENI): Historia y fundamentos teóricos de su validación. Un acercamiento práctico a su uso y valor diagnóstico. Revista Neuropsicología, Neuropsiquiatría y Neurociencias, 2014; 14; 68-95.

18. Barros MC, Ison MS. Conductas problemas infantiles: indicadores evolutivos $y$ emocionales en el dibujo de la figura humana. Interamerican J Psychol 2002;36:279-98.

19. Levin, M y Contini de González N. ¿Por qué hay niños que no aprenden a escribir? Aportes de la evaluación psicológica / Why there are children who do not learn to write? Contributions of the psychological evaluation Rev Med Tucumán 2004;10:6984.

20. Koppitz, E. Psychological evaluation of children's human figure drawings. New York: Grune \& Stratton, 1968.

21. Sohlberg MM, Mateer, CA. Improving attention and managing attentional problems. Adapting rehabilitation techniques to adults with ADD. Ann N Y Acad Sci 2001;931:359-75.
22. Meichenbaum, D. Changing conceptions of cognitive behavior modification: Retrospect and prospect. J Consult Clin Psychol 1993;61:202-4. Doi:10.1037/0022006X.61.2.202

23. Cicerone KD. Commentary: The validity of cognitive rehabilitation. J Head Trauma Rehab 1999;14:316-21. discussion 322-4.

24. Cicerone KD, Dahlberg C, Kalmar K, Langenbahn DM, Malec JF, Bergquist TF, et al. Evidence-based cognitive rehabilitation: Recommendations for clinical practice. Arch Phys Med Rehabil 2000;81:1596-615.

25. Catroppa C, Anderson V. Recovery in memory function, and its relationship to academic success, at 24 months following pediatric TBI. Child Neuropsychol 2007;13:240-6.

26. Cicerone KD. Langenbahn DM, Braden C, Malec JF, Kalmar K, Fraas M, et al. Evidence-based cognitive rehabilitation: Updated review of the literature from 2003 through 2008. Arch Phys Med Rehabil 2011;92:519-30. Doi: 10.1016/j. apmr.2010.11.015.

27. Cicerone KD, Dahlberg C, Malec JF, Langenbahn DM, Felicetti T, Kneipp S, et al. Evidence-based cognitive rehabilitation: Updated review of the literature from 1998 through 2002. Arch Phys Med Rehabil 2005;86:1681-92. Doi: 10.1016/j. apmr.2013.04.003.

28. Nazemi KJ, Butler RW. Neuropsychological rehabilitation for survivors of childhood and adolescent brain tumors: A view of the past and a vision for a promising future. J Pediatr Rehabil Med 2011;4:37-46. Doi: 10.3233/PRM-2011-0151.

29. Garcia-Perez A, Narbona-Garcia J, Sierrasesumaga L, Aguirre-Ventallo M., CalvoManuel F. Neuropsychological outcome of children after radiotherapy for intracranial tumours. Dev Med Child Neurol 1993;35:139-48 